\title{
Review Article \\ Evaluation of Antiviral Therapy Performed after Curative Therapy in Patients with HBV-Related Hepatocellular Carcinoma: An Updated Meta-Analysis
}

\author{
Peng Yuan, Peng Chen, and Yeben Qian \\ Department of Hepatobiliary Surgery, The First Affiliated Hospital of Anhui Medical University, Hefei 230032, China \\ Correspondence should be addressed to Yeben Qian; qianyeben@hotmail.com
}

Received 22 July 2015; Accepted 1 September 2015

Copyright (C) 2016 Peng Yuan et al. This is an open access article distributed under the Creative Commons Attribution License, which permits unrestricted use, distribution, and reproduction in any medium, provided the original work is properly cited.

\begin{abstract}
Background. The long-term prognosis after curative therapy for hepatitis B virus- (HBV-) related hepatocellular carcinoma (HCC) remains unsatisfactory due to the high incidence of recurrence. The effect of treatment with nucleotide analogues (NAs) in patients with HBV-related HCC after curative therapy remains unclear. Objective. To assess the impact of using NAs after curative therapy. Method. A computerized literature search was performed; eligible studies were identified from databases. The pooled risk ratios (RRs) and 95\% CIs were calculated using Review Manager 5.3. Result. The meta-analysis included a total of 15 studies with 8060 patients. The one-year and three-year recurrence (one-year recurrence: RR 0.41 [95\% CI 0.28 to 0.61 ]; $P<0.00001$; three-year recurrence: RR 0.63 [95\% CI 0.43 to 0.94]; $P=0.001$ ) and the one-, three-, and five-year overall survival (OS) and diseasefree survival (DFS) were significantly better in the treatment group. Conclusion. NAs can reduce the recurrence and improve the prognosis of HBV-related HCC after curative therapy.
\end{abstract}

\section{Introduction}

Hepatocellular carcinoma (HCC) is the fifth most common cancer in the world and the third leading cause of cancerrelated death $[1,2]$. It is a common malignancy worldwide, but especially in Asia, due to the endemic status of chronic hepatitis B [3], which is responsible for HBV-related HCC (HBV-HCC). In patients with HBV infection who develop HCC, treatment for one condition often influences the other condition: for example, treatment for HCC may affect viral replication, whereas treatment for chronic HBV infection may influence the clinical outcome of HCC [4]. Curative therapies including liver transplantation, hepatectomy, and local ablative therapy such as radiofrequency ablation are used to treat HCC. With the advances in surgery, the 5year survival rate after curative therapy has reached 50\% [5]. However, this is not satisfactory because the posttreatment recurrence rate is high [6]. Relapse is reported to occur in up to $70 \%$ of patients within 5 years of curative treatment [7]. Recent studies have shown that HBV viral replication plays an important role in tumor recurrence and might influence postoperative survival [8-12].
The results of 6 prospective studies, 11 retrospective studies, and 5 meta-analyses have shown that nucleotide analogues (NAs) might improve the overall survival (OS) $[4,8,10,13-30]$. However, only a few studies have shown that NAs decrease the recurrence rate or improve recurrencefree survival (RFS) $[17,19,20,24-26,29]$. Therefore, it is still not clear whether NAs can reduce the recurrence of HCC or improve RFS. In order to [13-25] investigate this, we performed a meta-analysis to sum up the current evidence from the literature, to determine the role that NAs play in the treatment of patients who have undergone curative therapy.

\section{Method}

2.1. Study Selection. A computerized search was performed in January 2015 by searching Medline, OVID, Cochrane, Embase, and Chinese Biological Medicine (CBM) from the time of inception to January 2015. The search was performed using the main keywords "HCC", "liver cancer", "hepatocellular carcinoma", "HBV", "hepatitis B", "resection", "hepatectomy", "curative therapy", "nucleotide", "entecavir", "lamivudine", "adefovir", "telbivudine", "recurrence", and 


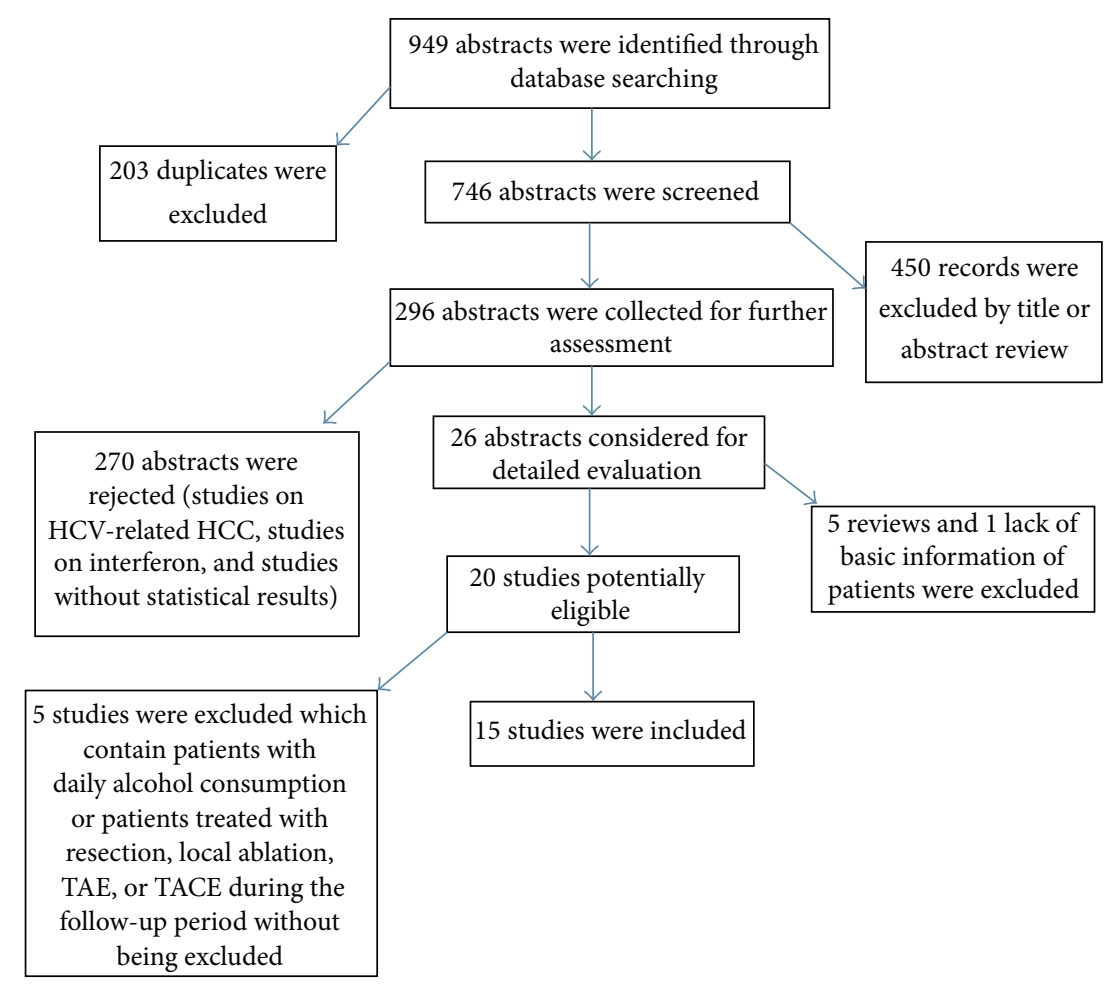

FIGURE 1: Flow chart depicting the study selection process: 15 studies were included in this meta-analysis.

"prognosis". Curative therapy was defined as any method that could remove evident tumors completely with no remnant tumor tissue found within 4 weeks after the treatment.

We included randomized controlled trials (RCTs), prospective cohort trials, and retrospective cohort trails that (1) included patients with confirmed chronic HBV infection; (2) included patients with confirmed HCC as determined by histopathological examination or radiological examination in addition to alpha-fetoprotein; (3) used postoperative antiviral treatment; (4) did not administer any treatment for control patients and those with HCC recurrence and/or mortality that developed during the follow-up period before the patients were excluded from the trials; and (5) included the OS, disease-free survival (DFS), or recurrence rate (one of the three results) in the follow-up data (studies that included RFS were also accepted as RFS is considered equivalent to DFS).

We excluded (1) studies on patients who received antiviral therapy before HCC diagnosis; (2) studies in which noncurative therapies including image-guided tumor ablation using chemical or thermal ablative techniques for unresectable tumors and transarterial chemoembolization (TACE) were performed; (3) studies that included patients coinfected with HCV, HDV, or HIV; (4) studies on patients with daily alcohol consumption; and (5) studies on patients with drug abuse.

2.2. Data Extraction and Validity Assessment. The methodological qualities and relevance of the studies were assessed by two reviewers (Peng Yuan and Peng Chen) independently. The following data were extracted by the two reviewers: type of study, year of study, sample size, characteristics of the patients, follow-up duration, tumor stage, HBV DNA level, Child-Pugh grade, ALT level, cirrhosis rate, tumor characteristics, types of curative treatment, tumor recurrence rate, DFS (or RFS), and OS. The quality of studies was assessed for RCT studies and cohort studies using the JADAD scale [31] and Newcastle-Ottawa scale [32], respectively (Table 1). If any discrepancies were noticed between the two reviewers in the process of data extraction and quality assessment, a third reviewer (Yeben Qian) made the final decision.

2.3. Statistical Analysis. RevMan version 5.3 was used to perform the statistical analyses. Relative risk (RR) was determined for each study. A random-effect model was used in this meta-analysis to calculate the overall effects estimates. Heterogeneity was assessed based on the $I^{2}$ value, which indicated the percentage of total variation across studies and $P<0.1$ was considered to indicate statistical significance. The funnel plot was used to evaluate publication biases.

\section{Results}

3.1. Study Selection and Characteristics of the Included Studies. The literature search yielded 949 abstracts. As showed in Figure 1, by excluding 203 duplicates and 450 irrelevant abstracts, 296 were collected for further evaluation, of which 26 abstracts were considered for detailed evaluation and the remaining 270 were rejected because they were on $\mathrm{HCV}$ related HCC or interferon therapy, they did not provide the statistical results required, or they were on patients who did 


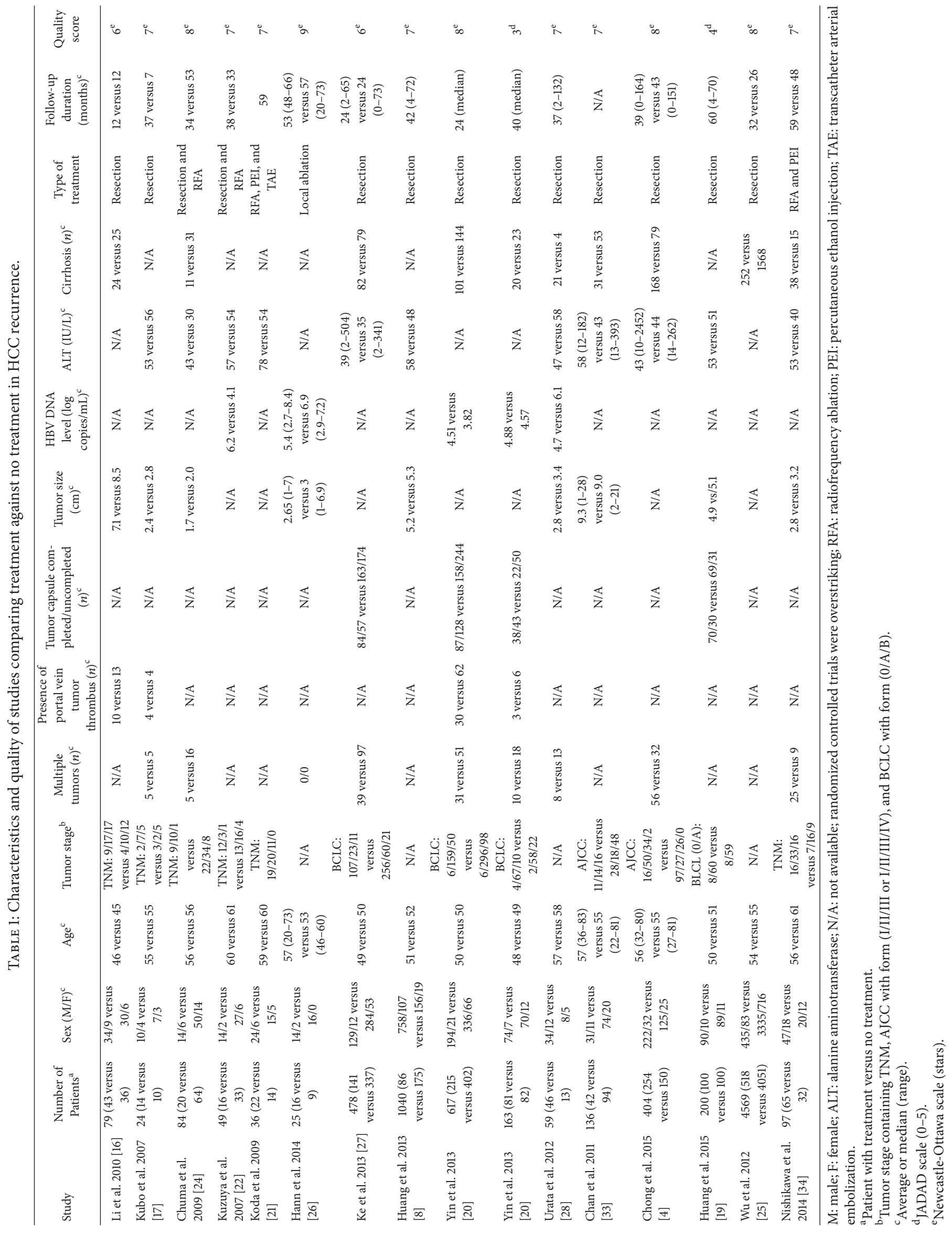


not receive curative therapy before the use of NAs. Among the 26 publications, five meta-analyses and one study that lacked basic information on patients were excluded, and five other studies were finally excluded because one of them included patients with daily alcohol consumption and the other four did not exclude patients who had undergone resection, local ablation, transarterial embolization (TAE), or TACE during the follow-up period. Finally, 15 studies were included in the meta-analysis, including two RCTs and 13 cohort studies (three prospective studies, nine retrospective studies, and one that had both prospective and retrospective samples). In the study by Yin et al. [20], a randomized controlled trial and a nonrandomized trial were performed, so we considered this as two individual studies in this meta-analysis.

This analysis included a total of 8060 patients, 2498 of whom were in the treatment group and 5562 of whom were in the control group (without NA treatment). The curative therapies performed included liver resection; local ablation; and all treatments which guaranteed that all macroscopically evident tumors were removed completely, tumor cells were not present along the parenchymal transection line (which was confirmed histologically), and computed tomography performed at least four weeks after surgery did not show any remnant tumor tissue. Baseline comparison was performed in all the 15 studies, and no significant difference was found between both groups in terms of age, gender ratio, ChildPugh grade, percentage of $\mathrm{HBeAg}$-positive patients, $\mathrm{HBV}$ DNA level, presence of cirrhosis, tumor stage, AFP level, and HCC treatment. Among the 15 studies included, nine studies used curative hepatectomy as the initial treatment for HCC while the other five studies included patients treated with radiofrequency ablation and other curative therapies. Four studies used single-nucleotide analogues as antiviral therapy (two used adefovir and two used lamivudine), and the other 11 studies initiated the treatment with lamivudine or entecavir and then switched to adefovir, telbivudine, or tenofovir disoproxil fumarate when drug resistance occurred or TMDD mutants were detected.

3.2. Antiviral Treatment and Recurrence. We pooled the data from 6 of 15 studies which reported the recurrence rate or cause of death of patients, so that we could calculate the number of patients with HCC recurrence. However, in $\mathrm{Wu}$ et al.s [25] study, a large number of patients who used drugs, including NSAIDs and statins, and patients with diabetes were not excluded from the retrospective cohorts. As the number of samples in which recurrence was analyzed was not sufficient, Wu et al's study was included and analyzed. We found that the one-year recurrence rate was significantly lower in the antiviral treatment group (94 of 665) than in the untreated group (971 of 4249), with the risk of recurrence reduced by $50 \%$ in the treatment group ( $R R=0.50,95 \%$ $\mathrm{CI}=0.36-0.68, P<0.0001$, Figure 2(a)). The three-year recurrence rate was also lower in the antiviral treatment group (239 of 665) than in the untreated group (1921 of 4249). The risk of recurrence was found to be reduced by $30 \%(\mathrm{RR}=$ $0.70,95 \% \mathrm{CI}=0.56-0.87, P=0.001$, Figure $2(\mathrm{~b})$ ) on pooling the data from all the six studies. There was no significant heterogeneity in the 1-year while there was heterogeneity in 3-year recurrence rate (1-year recurrence: $I^{2}=22 \%$ and $P=$ 0.27 ; 3 -year recurrence: $I^{2}=51 \%$ and $\left.P=0.07\right)$. The funnel plot for evaluation of publication bias is shown in Figure 5.

When Wu et al's study was not included, the 1-year recurrence rate was significantly lower in the antiviral treatment group ( 25 of 147) than in the untreated group (68 of 198), with the risk of recurrence reduced by $59 \%$ in the treatment group $(\mathrm{RR}=0.41,95 \% \mathrm{CI}=0.28-0.61, P<0.00001$, Figure $2(\mathrm{c}))$. Further, the 3 -year recurrence rate was also lower in the antiviral treatment group (71 of 147) than in the untreated group (136 of 198). The risk of recurrence was found to be reduced by $37 \%(\mathrm{RR}=0.63,95 \% \mathrm{CI}=0.43-0.94, P=$ 0.03 , Figure $2(d)$ ) on pooling the data from the five studies (after excluding Wu et al.s study). There was no significant heterogeneity in the 1-year while there was heterogeneity in 3-year recurrence rate (1-year recurrence: $I^{2}=0 \%$ and $P=$ 0.45 ; 3 -year recurrence: $I^{2}=61 \%$ and $P=0.04$ ).

3.3. Antiviral Treatment and OS. On pooling the data from 10 of 15 studies, the 1-year OS was found to be higher in the antiviral treatment group (728 of 819) than in the untreated group (701 of 900), with an improvement of $11 \%$ in the treatment group $(\mathrm{RR}=1.11,95 \% \mathrm{CI}=1.05-1.16, P<0.0001$, Figure 3(a)). Further, the 3-year and 5-year OS values were significantly higher in the antiviral treatment group (402/551 and $496 / 674$, resp.) than in the untreated group (407/747 and $394 / 749$, resp.), which corresponded to an improvement of $28 \%(\mathrm{RR}=1.28 ; 95 \% \mathrm{CI}=1.14-1.44, P<0.0001$, Figure $3(\mathrm{~b}))$ and $40 \%(\mathrm{RR}=1.40,95 \% \mathrm{CI}=1.24-1.58, P<0.00001$, Figure $3(\mathrm{c})$ ), respectively. Heterogeneity was observed in the 3-year and 5-year OS, but no significant heterogeneity observed in 1-year OS (1-year OS: $I^{2}=25 \%$ and $P=0.22$; 3-year OS: $I^{2}=45 \%$ and $P=0.08$; 5 -year OS: $I^{2}=45 \%$ and $P=0.08)$.

3.4. Antiviral Treatment and DFS/RFS. On pooling the data from 12 of 15 studies, we found that the 1-year DFS was higher in the antiviral treatment group (714 of 1093) than in the untreated group (743 of 1279), and DFS improved by $17 \%$ with antiviral treatment $(\mathrm{RR}=1.17,95 \% \mathrm{CI}=1.04-1.31, P<0.008$, Figure 4(a)). Further, the 3-year and 5-year DFS values were significantly higher in the antiviral treatment group (555/1245 and $232 / 531$, resp.) than in the untreated group (156/510 and $122 / 389$, resp.), which means that DFS improved by $52 \%$ (RR $=1.52,95 \% \mathrm{CI}=1.21-1.91, P=0.0003$, Figure $4(\mathrm{~b}))$ and $50 \%$ $(\mathrm{RR}=1.50,95 \% \mathrm{CI}=1.12-2.00, P=0.006$, Figure 4(c) $)$, respectively, with antiviral treatment. Low heterogeneity was observed in the 3-year and 5-year DFS (3-year DFS: $I^{2}=37 \%$ and $P=0.12$; 5-year DFS: $I^{2}=39 \%$ and $\left.P=0.13\right)$, but median heterogeneity was observed in the 1-year DFS $\left(I^{2}=\right.$ $60 \%, P=0.004)$.

3.5. Subgroup Analysis with RCTs Excluded and High Score Studies. In subgroups, we pooled data from all observational studies and studies with Newcastle-Ottawa no less than 8 stars, respectively. The results still showed significant difference between NAs group and untreated group. The result was shown in Table 2. 


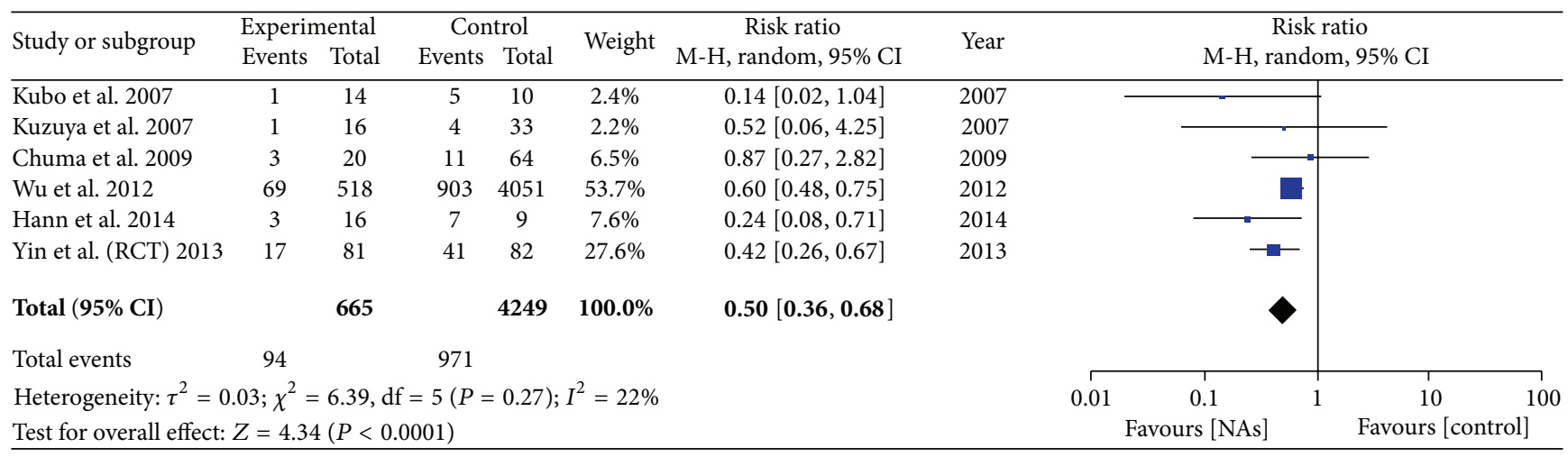

(a)

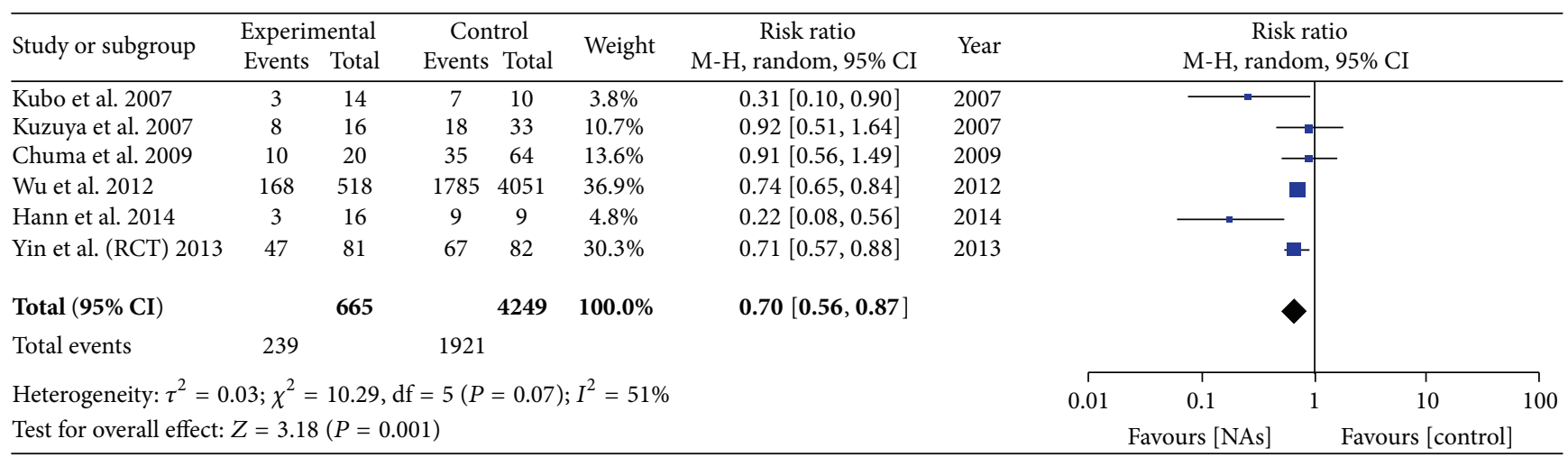

(b)



(c)

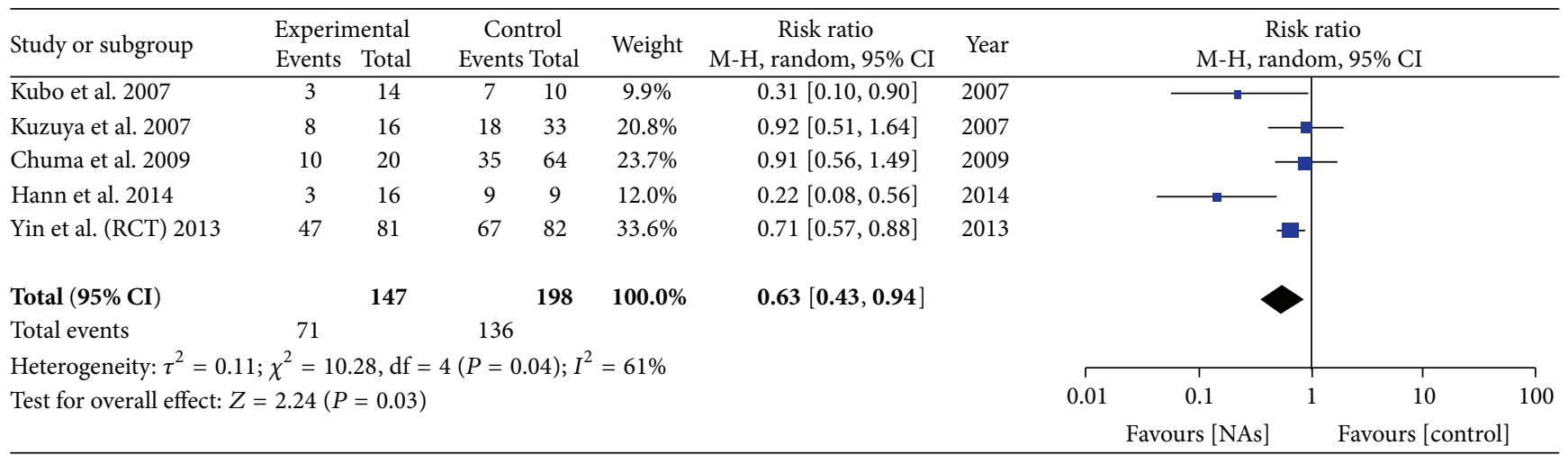

(d)

Figure 2: (a) Forest plot showing the impact of NAs on the 1-year recurrence rate without Wu et al's study. (b) Forest plot showing the impact of NAs on the 3-year recurrence rate without Wu et al.s study. (c) Forest plot showing the impact of NAs on the 1-year recurrence rate with Wu et al.s study. (d) Forest plot showing the impact of NAs on the 3-year recurrence rate with Wu et al.'s study. 


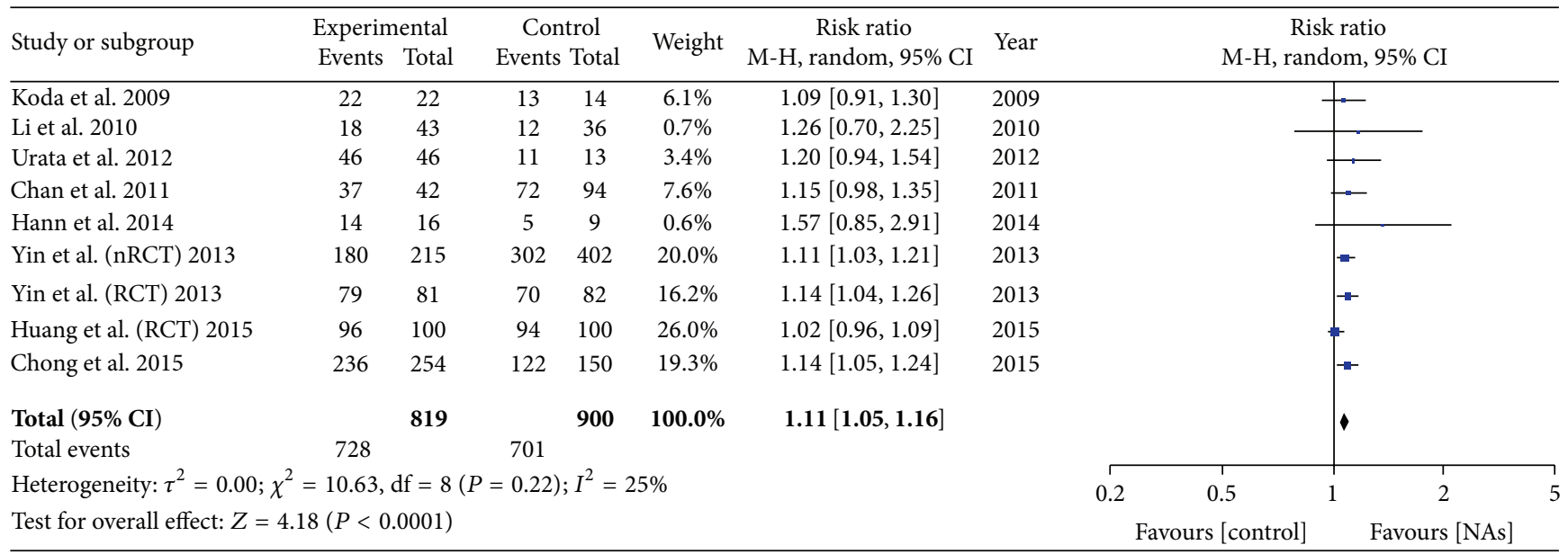

(a)

\begin{tabular}{|c|c|c|c|c|c|c|c|c|c|c|c|c|}
\hline \multirow{2}{*}{$\frac{\text { Study or subgroup }}{\text { Kuzuya et al. } 2007}$} & \multicolumn{2}{|c|}{ Experimental } & \multicolumn{2}{|c|}{$\begin{array}{c}\text { Control } \\
\text { Events Total }\end{array}$} & Weight & $\begin{array}{c}\text { Risk ratio } \\
\text { M-H, random, } 95 \% \text { CI }\end{array}$ & Year & \multicolumn{5}{|c|}{$\begin{array}{c}\text { Risk ratio } \\
\mathrm{M}-\mathrm{H} \text {, random, } 95 \% \mathrm{CI}\end{array}$} \\
\hline & 16 & 16 & 27 & 33 & $17.4 \%$ & $1.20[1.00,1.44]$ & 2007 & & & - & & \\
\hline Koda et al. 2009 & 17 & 22 & 8 & 14 & $4.5 \%$ & $1.35[0.81,2.25]$ & 2009 & & & & - & \\
\hline Chan et al. 2011 & 33 & 42 & 45 & 94 & $11.9 \%$ & $1.64[1.26,2.14]$ & 2011 & & & & - & \\
\hline Hann et al. 2014 & 13 & 16 & 1 & 9 & $0.4 \%$ & $7.31[1.14,47.11]$ & 2014 & & & & & \\
\hline Yin et al. (RCT) 2013 & 65 & 81 & 43 & 82 & $13.7 \%$ & $1.53[1.21,1.93]$ & 2013 & & & & $=$ & \\
\hline Yin et al. (nRCT) 2013 & 128 & 215 & 205 & 402 & $20.6 \%$ & $1.17[1.01,1.35]$ & 2013 & & & $=$ & & \\
\hline Nishikawa 2014 & 57 & 65 & 24 & 32 & $14.6 \%$ & $1.17[0.94,1.46]$ & 2014 & & & $=-$ & & \\
\hline Huang et al. (RCT) 2015 & 73 & 94 & 54 & 81 & $17.0 \%$ & $1.16[0.96,1.41]$ & 2015 & & & $=$ & & \\
\hline Total $(95 \% \mathrm{CI})$ & & 551 & & 747 & $100.0 \%$ & $1.28[1.14,1.44]$ & & & & $\Delta$ & & \\
\hline \multicolumn{13}{|c|}{$\begin{array}{l}\text { Total events } \\
\text { Heterogeneity: } \tau^{2}=0.01 ; \chi^{2}=12.79, \mathrm{df}=7(P=0.08) ; I^{2}=45 \%\end{array}$} \\
\hline \multicolumn{8}{|c|}{ Test for overall effect: $Z=4.24(P<0.0001)$} & 0.01 & 0.1 & 1 & 10 & 100 \\
\hline & & & & & & & & \multicolumn{5}{|c|}{ Favours [control] } \\
\hline
\end{tabular}

(b)

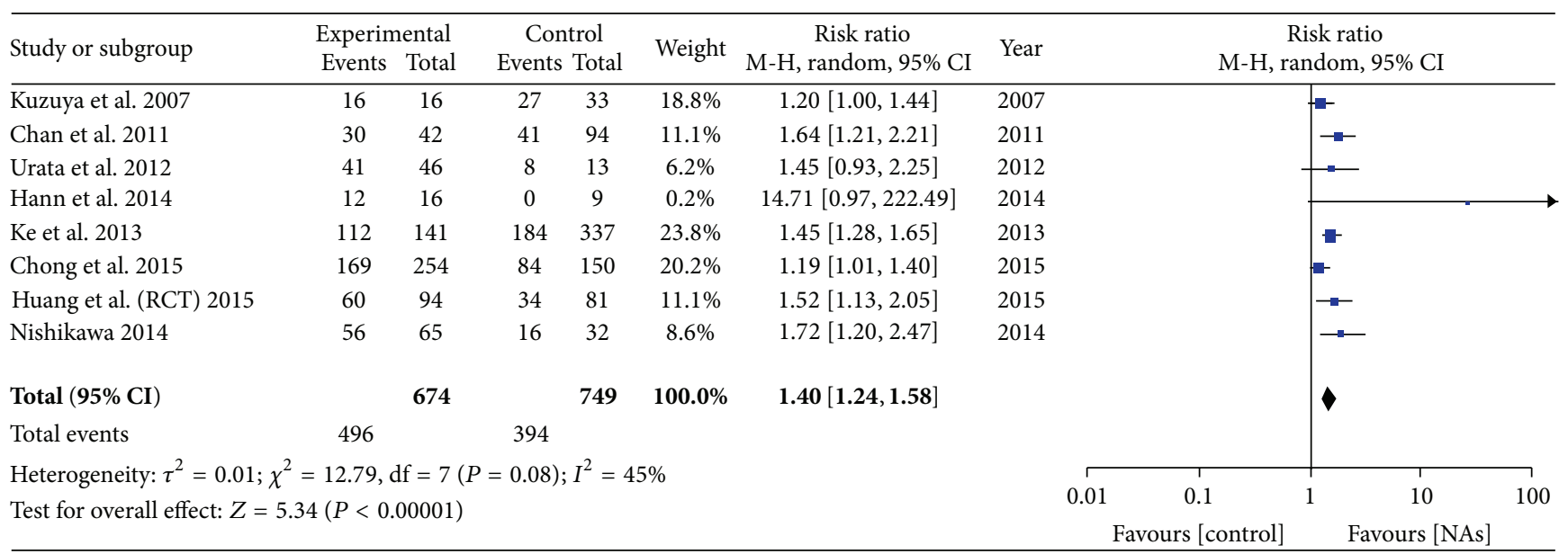

(c)

FIGURE 3: (a) Forest plot showing the impact of NAs on the 1-year OS. (b) Forest plot showing the impact of NAs on the 3-year OS. (c) Forest plot showing the impact of NAs on the 5-year OS. 


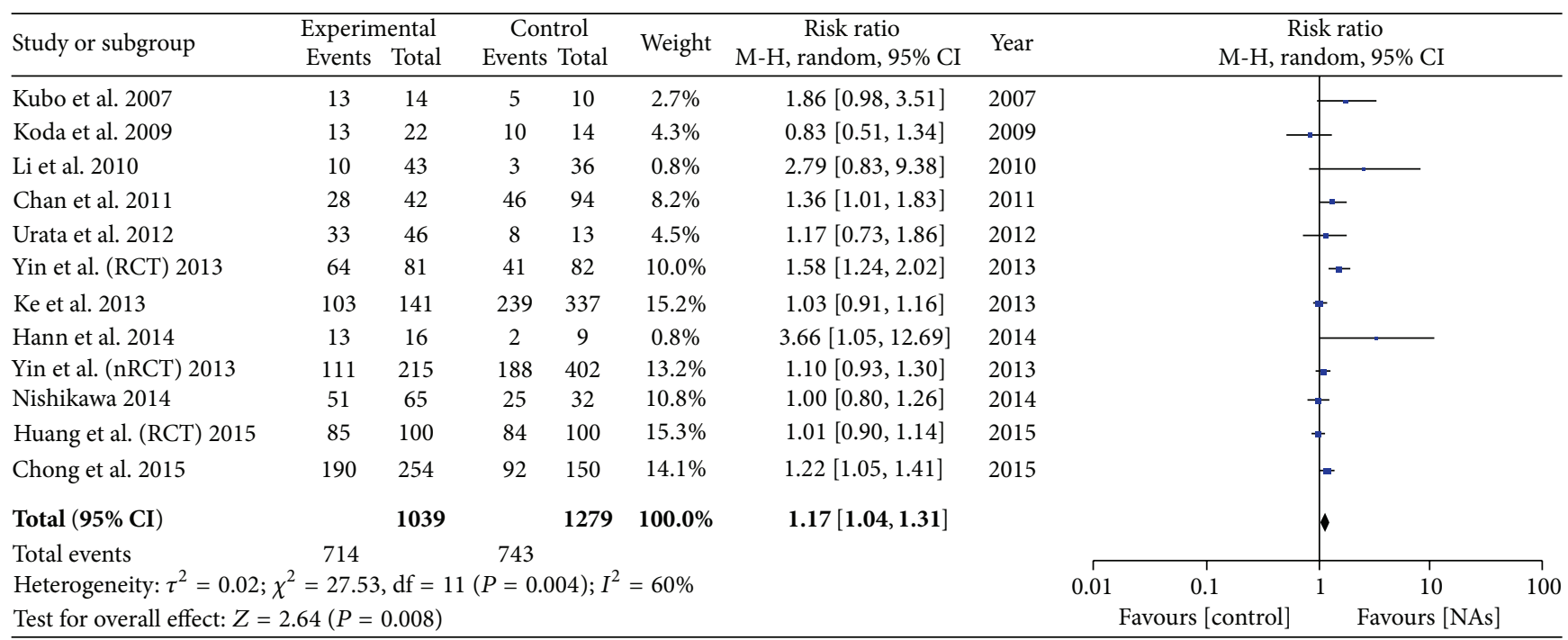

(a)

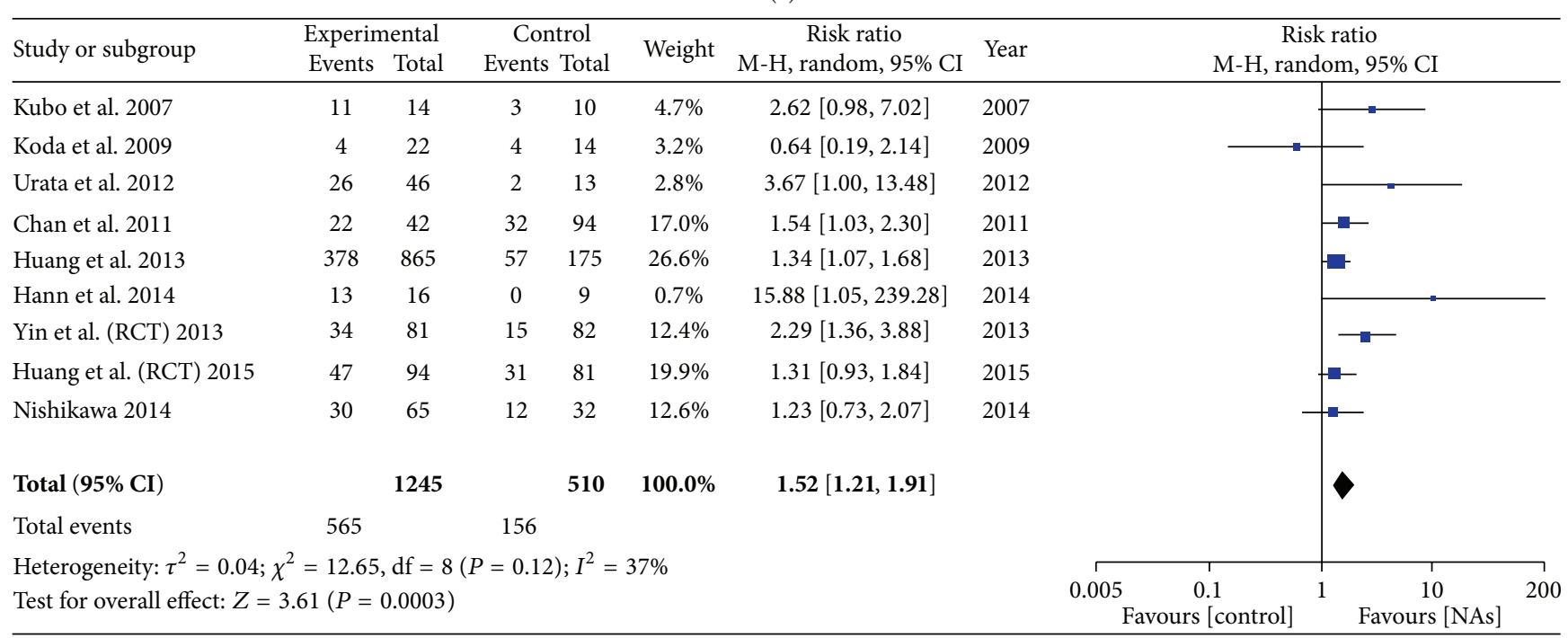

(b)

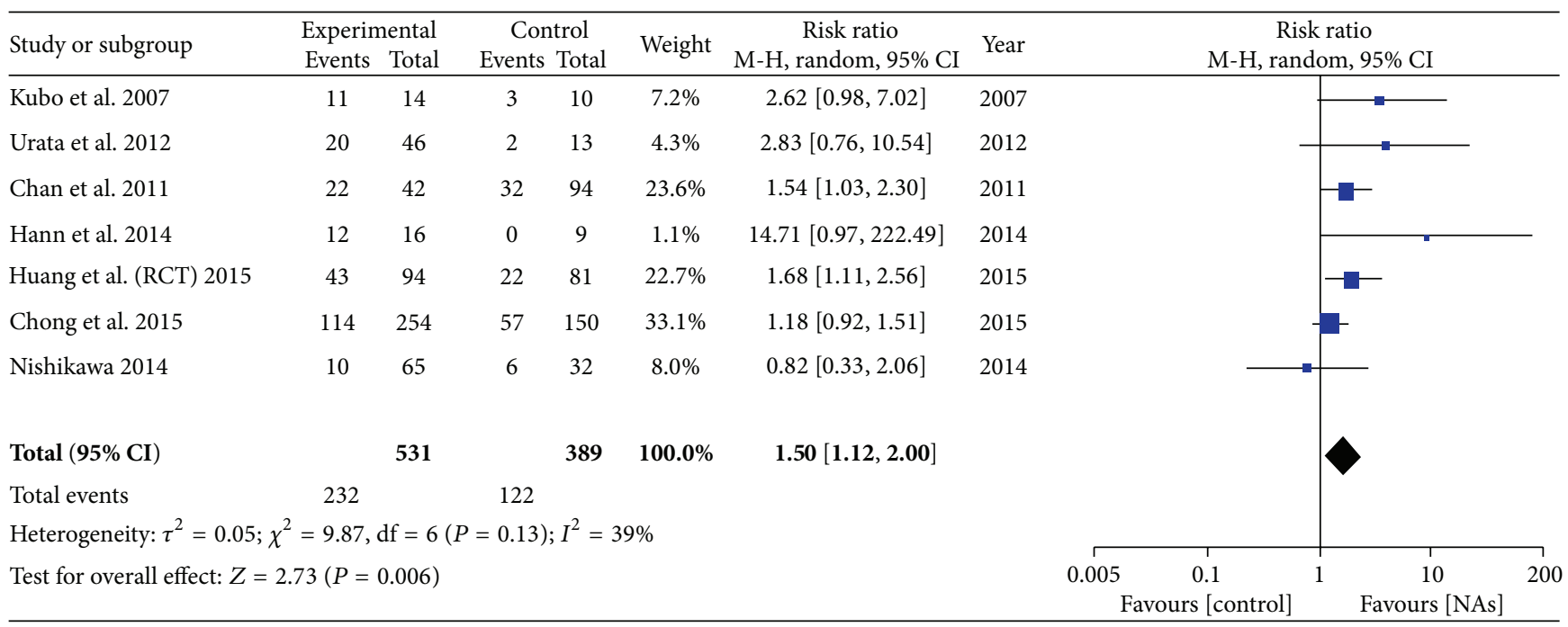

(c)

FIgURE 4: (a) Forest plot showing the impact of NAs on the 1-year DFS. (b) Forest plot showing the impact of NAs on the 3-year DFS. (c) Forest plot showing the impact of NAs on the 5-year DFS. 







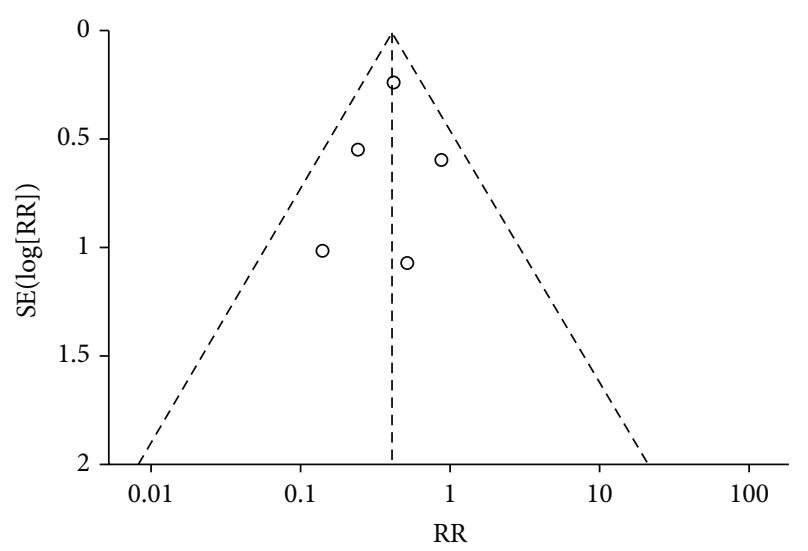

FIGURE 5: Funnel plot analysis of publication bias. The outcome impact of NAs on the 1-year HCC recurrence rate.

\section{Discussion}

Analysis of the data from the 15 studies examined provided significant evidence of the benefits of using NAs, as the use of NAs was associated with a decreased HCC recurrence rate after curative therapy and improved RFS, especially long-term survival (more than one year). HBV-related HCC accounts for the majority of HCC cases in the Asia-Pacific region: $80-90 \%$ of patients with $\mathrm{HCC}$ have chronic $\mathrm{HBV}$ infection [26]. With the advances in surgery, the survival rate of HBV-related HCC after curative therapy has improved, but the recurrence rate is still high and severely affects patient survival. The mechanism of HCC recurrence is still unclear; however, the results of different types of studies (RCTs, cohort studies, and meta-analyses) have indicated that a high viral load of HBV before or after curative treatment may increase the recurrence rate of patients after curative surgery for HCC and reduce OS and DFS [13, 20, 27, 28].

The use of NAs, including lamivudine, adefovir dipivoxil, and entecavir, has been proved to be beneficial in preventing progression to cirrhosis and delaying the development of HCC in patients with chronic HBV infection [33, 3539]. Compared with other adjuvant therapies, especially interferon therapy, NA therapy has been shown to be safer and better tolerated; it has been shown that NA therapy can reduce the risk of hepatic decompensation with lifethreatening complications, including hepatic encephalopathy, icterus, ascites, and variceal bleeding, in patients with advanced liver disease [40]. However, the effects of NAs in patients who have undergone initial curative therapy are unclear for many reasons. (1) One of the reasons is the lack of enough samples, as it is difficult to perform RCTs for ethical reasons. (2) Another reason is the emergence of drug resistance, as evidenced by the rate of lamivudine resistance, which has been reported to be $14-39 \%[16,17,21$, 22]. Although newer NAs that are currently recommended by international guidelines may provide better viral suppression and potentially even better long-term outcomes [15, 41-43], in rural areas and underdeveloped regions, lamivudine is the only drug that patients wish to take for economic reasons. (3) Yet another reason is that it is difficult to eliminate differences between surgeons and hospitals with regard to the treatment strategies. (4) Finally, as patients with HBVrelated HCC often have other serious diseases such as peptic ulcer, hypertension, and renal dysfunctions, which would have an influence on the effect of NAs, it would be difficult to understand the true effect of NAs in these patients.

Our meta-analysis tried to eliminate the abovementioned limitations as much as possible and come to a relatively objective conclusion. The results showed that the use of NAs to treat patients after curative therapy for HBV-related HCC might reduce recurrence and improve the OS and DFS or RFS.

The short-term recurrence rate (1-year and 3-year) was obviously higher in the untreated groups, but we were unable to determine the recurrence rate over a longer period because of the unavailability of raw data and detailed information on the causes of death. For example, in cases where death was caused by liver failure, it would be very difficult to separate the role of the tumor from the role of liver failure and therefore determine the exact recurrence rate. Therefore, we only included studies in which the recurrence rates were already calculated. OS was higher in the treatment groups at 1,3 , and 5 years, but the 3-and 5-year OS values were more obviously higher in the treatment group. This finding could be attributable to the advances in surgical treatment for HBV-related HCC and overall improvement in short-term prognosis. Analysis of DFS revealed similar results.

As our meta-analysis revealed, NAs can reduce the recurrence of HCC and improve DFS after curative therapy, which is in contrast to the conclusion of some studies that NAs do not reduce the recurrence rate $[4,16,18,22,23,27]$. Furthermore, we tried to eliminate the effects of NSAIDs by excluding studies in which HCC patients were given these drugs, because latest research has revealed that NSAIDs might also reduce the recurrence of HCC after curative therapy [44]. Further, the 1-year and 3-year recurrence rates considerably improved with or without the inclusion of $\mathrm{Wu}$ et al.s study.

There are several limitations in our meta-analysis: (1) In the 11 cohort studies included, the treatment and notreatment groups were not matched. (2) The cut-off value of serum HBV DNA in the trials differs: although the majority of the references set the cut-off value as $10^{4}$ copies $/ \mathrm{mL}$, the cut-off value was 500 copies $/ \mathrm{mL}$ in Yin et al.'s study [20], and four other studies $[4,8,18,26]$ included samples in which the serum HBV level was lower than $10^{4}$ copies $/ \mathrm{mL}$. (3) The heterogeneity of the baseline characteristics, such as tumor size, number of tumors, and cirrhosis rate before initial HCC treatment, may cause a potential bias. (4) In prospective studies, it was difficult to exclude samples in which the patients were not followed up, which may have caused a potential bias. (5) Some of the studies did not exclude patients who used NSAIDs during the follow-up period, so we need to further investigate this by eliminating the effect of NSAIDs. (6) Although liver transplantation is one of most important curative therapies on HCC, unfortunately no applicable study on using NAs after liver transplantation was included in our meta-analysis. 
In conclusion, antiviral therapy with NAs has potential benefits with regard to reducing the recurrence rate and improving the OS and DFS of patients with HBV-related HCC after curative therapy. Therefore, NA treatment should be recommended for individuals with HBV-related HCC, provided their serum HBV level and general health status are suitable for NA use.

\section{Competing Interests}

The authors declare that there are no competing interests regarding the publication of this paper.

\section{Authors' Contributions}

Peng Yuan and Peng Chen contributed equally to this work.

\section{Acknowledgments}

The authors would like to thank the First Affiliated Hospital of Anhui Medical University, the Department of Hepatobiliary Surgery, Hefei, Anhui, China, and all the reviewers for their hard work.

\section{References}

[1] A. I. Gomaa, S. A. Khan, M. B. Toledano, I. Waked, and S. D. Taylor-Robinson, "Hepatocellular carcinoma: epidemiology, risk factors and pathogenesis," World Journal of Gastroenterology, vol. 14, no. 27, pp. 4300-4308, 2008.

[2] J. M. Llovet, A. Burroughs, and J. Bruix, "Hepatocellular carcinoma," The Lancet, vol. 362, no. 9399, pp. 1907-1917, 2003.

[3] H. L.-Y. Chan and J. J.-Y. Sung, "Hepatocellular carcinoma and hepatitis B virus," Seminars in Liver Disease, vol. 26, no. 2, pp. 153-161, 2006.

[4] C. Chong, G. Wong, V. Wong et al., "Antiviral therapy improves post-hepatectomy survival in patients with hepatitis B virusrelated hepatocellular carcinoma: a prospective-retrospective study," Alimentary Pharmacology and Therapeutics, vol. 41, no. 2, pp. 199-208, 2015.

[5] J. Wong, K. F. Lee, P. S. F. Lee et al., "Radiofrequency ablation for 110 malignant liver tumours: preliminary results on percutaneous and surgical approaches," Asian Journal of Surgery, vol. 32, no. 1, pp. 13-20, 2009.

[6] T. Toyama, N. Hiramatsu, T. Yakushijin et al., "A new prognostic system for hepatocellular carcinoma including recurrent cases: a study of 861 patients in a single institution," Journal of Clinical Gastroenterology, vol. 42, no. 3, pp. 317-322, 2008.

[7] J. M. Llovet, M. Schwartz, and V. Mazzaferro, "Resection and liver transplantation for hepatocellular carcinoma," Seminars in Liver Diseases, vol. 25, no. 2, pp. 181-200, 2005.

[8] G. Huang, E. C. H. Lai, W. Y. Lau et al., "Posthepatectomy HBV reactivation in hepatitis B-Related hepatocellular carcinoma influences postoperative survival in patients with preoperative low HBV-DNA levels," Annals of Surgery, vol. 257, no. 3, pp. 490-505, 2013.

[9] H.-C. Sun, W. Zhang, L.-X. Qin et al., "Positive serum hepatitis $\mathrm{B}$ e antigen is associated with higher risk of early recurrence and poorer survival in patients after curative resection of hepatitis
B-related hepatocellular carcinoma," Journal of Hepatology, vol. 47, no. 5, pp. 684-690, 2007.

[10] I. F. N. Hung, R. T. P. Poon, C.-L. Lai, J. Fung, S.-T. Fan, and M.-F. Yuen, "Recurrence of hepatitis b-related hepatocellular carcinoma is associated with high viral load at the time of resection," American Journal of Gastroenterology, vol. 103, no. 7, pp. 1663-1673, 2008.

[11] Y. Hoshida, "Risk of recurrence in hepatitis B-related hepatocellular carcinoma: impact of viral load in late recurrence," Journal of Hepatology, vol. 51, no. 5, pp. 842-844, 2009.

[12] H. J. An, J. W. Jang, S. H. Bae et al., "Sustained low hepatitis $B$ viral load predicts good outcome after curative resection in patients with hepatocellular carcinoma," Journal of Gastroenterology and Hepatology, vol. 25, no. 12, pp. 1876-1882, 2010.

[13] Y. Zhou, Z. Zhang, Y. Zhao, L. Wu, and B. Li, "Antiviral therapy decreases recurrence of hepatitis B virus-related hepatocellular carcinoma after curative resection: a meta-analysis," World Journal of Surgery, vol. 38, no. 9, pp. 2395-2402, 2014.

[14] Y. Ke, L. Wang, L.-Q. Li, and J.-H. Zhong, "Nucleos(t)ide analogues to treat hepatitis B virus-related hepatocellular carcinoma after radical resection," World Journal of Hepatology, vol. 6, no. 9, pp. 652-659, 2014.

[15] J. S.-W. Wong, G. L.-H. Wong, K. K.-F. Tsoi et al., "Metaanalysis: the efficacy of anti-viral therapy in prevention of recurrence after curative treatment of chronic hepatitis Brelated hepatocellular carcinoma," Alimentary Pharmacology and Therapeutics, vol. 33, no. 10, pp. 1104-1112, 2011.

[16] N. Li, E. C. H. Lai, J. Shi et al., "A comparative study of antiviral therapy after resection of hepatocellular carcinoma in the immune-active phase of hepatitis B virus infection," Annals of Surgical Oncology, vol. 17, no. 1, pp. 179-185, 2010.

[17] S. Kubo, H. Tanaka, S. Takemura et al., "Effects of lamivudine on outcome after liver resection for hepatocellular carcinoma in patients with active replication of hepatitis B virus," Hepatology Research, vol. 37, no. 2, pp. 94-100, 2007.

[18] C.-Y. Piao, S.-I. Fujioka, Y. Iwasaki et al., "Lamivudine treatment in patients with HBV-related hepatocellular carcinoma-using an untreated, matched control cohort," Acta Medica Okayama, vol. 59, no. 5, pp. 217-224, 2005.

[19] G. Huang, W. Y. Lau, Z.-G. Wang et al., "Antiviral therapy improves postoperative survival in patients with hepatocellular carcinoma: a randomized controlled trial," Annals of Surgery, vol. 261, no. 1, pp. 56-66, 2015.

[20] J. Yin, N. Li, Y. Han et al., "Effect of antiviral treatment with nucleotide/nucleoside analogs on postoperative prognosis of hepatitis B virus-related hepatocellular carcinoma: a two-stage longitudinal clinical study," Journal of Clinical Oncology, vol. 31, no. 29, pp. 3647-3655, 2013.

[21] M. Koda, T. Nagahara, T. Matono et al., "Nucleotide analogs for patients with HBV-related hepatocellular carcinoma increase the survival rate through improved liver function," Internal Medicine, vol. 48, no. 1, pp. 11-17, 2009.

[22] T. Kuzuya, Y. Katano, T. Kumada et al., "Efficacy of antiviral therapy with lamivudine after initial treatment for hepatitis B virus-related hepatocellular carcinoma," Journal of Gastroenterology and Hepatology, vol. 22, no. 11, pp. 1929-1935, 2007.

[23] H. Yoshida, H. Yoshida, E. Goto et al., "Safety and efficacy of lamivudine after radiofrequency ablation in patients with hepatitis B virus-related hepatocellular carcinoma," Hepatology International, vol. 2, no. 1, pp. 89-94, 2008.

[24] M. Chuma, S. Hige, T. Kamiyama et al., "The influence of hepatitis B DNA level and antiviral therapy on recurrence 
after initial curative treatment in patients with hepatocellular carcinoma," Journal of Gastroenterology, vol. 44, no. 9, pp. 991999, 2009.

[25] C.-Y. Wu, Y.-J. Chen, H. J. Ho et al., "Association between nucleoside analogues and risk of hepatitis B virus-related hepatocellular carcinoma recurrence following liver resection," The Journal of the American Medical Association, vol. 308, no. 18, pp. 1906-1914, 2012.

[26] H.-W. Hann, R. Coben, D. Brown et al., "A long-term study of the effects of antiviral therapy on survival of patients with HBVassociated hepatocellular carcinoma (HCC) following local tumor ablation," Cancer Medicine, vol. 3, no. 2, pp. 390-396, 2014.

[27] Y. Ke, L. Ma, X.-M. You et al., "Antiviral therapy for hepatitis $B$ virus-related hepatocellular carcinoma after radical hepatectomy," Cancer Biology and Medicine, vol. 10, no. 3, pp. 158-164, 2013.

[28] Y. Urata, S. Kubo, S. Takemura et al., "Effects of antiviral therapy on long-term outcome after liver resection for hepatitis B virusrelated hepatocellular carcinoma," Journal of Hepato-BiliaryPancreatic Sciences, vol. 19, no. 6, pp. 685-696, 2012.

[29] B. W. Xia, Y. C. Zhang, J. Wang, F. H. Ding, and X. D. He, "Efficacy of antiviral therapy with nucleotide/nucleoside analogs after curative treatment for patients with hepatitis $B$ virus-related hepatocellular carcinoma: a systematic review and meta-analysis," Clinics and Research in Hepatology and Gastroenterology, vol. 39, no. 4, pp. 458-468, 2015.

[30] P. Sun, X. Dong, X. Cheng, Q. Hu, and Q. Zheng, "Nucleot(s)ide analogues for hepatitis B virus-related hepatocellular carcinoma after curative treatment: a systematic review and meta-analysis," PLoS ONE, vol. 9, no. 7, Article ID e102761, 2014.

[31] A. R. Jadad, R. A. Moore, D. Carroll et al., "Assessing the quality of reports of randomized clinical trials: is blinding necessary?" Controlled Clinical Trials, vol. 17, no. 1, pp. 1-12, 1996.

[32] G. A. Wells, S. B. O'Connell, D. Peterson et al., "The NewcastleOttawa Scale (NOS) for assessing the quality of nonrandomised studies in meta-analyses," 2014, http://www.ohri.ca/programs/ clinical_epidemiology/oxford.asp.

[33] A. C. Y. Chan, K. S. H. Chok, W. K. Yuen et al., "Impact of antiviral therapy on the survival of patients after major hepatectomy for hepatitis B virus-related hepatocellular carcinoma," Archives of Surgery, vol. 146, no. 6, pp. 675-681, 2011.

[34] H. Nishikawa, N. Nishijima, A. Arimoto et al., "Effect of nucleoside analog use in patients with hepatitis B virus-related hepatocellular carcinoma," Hepatology Research, vol. 44, no. 6, pp. 608-620, 2014.

[35] J.-C. Wu, Y.-H. Huang, G.-Y. Chau et al., "Risk factors for early and late recurrence in hepatitis B-related hepatocellular carcinoma," Journal of Hepatology, vol. 51, no. 5, pp. 890-897, 2009.

[36] S. Kubo, K. Hirohashi, H. Tanaka et al., "Effect of viral status on recurrence after liver resection for patients with hepatitis B virus-related hepatocellular carcinoma," Cancer, vol. 88, no. 5, pp. 1016-1024, 2000.

[37] J. L. Dienstag, R. D. Goldin, E. J. Heathcote et al., "Histological outcome during long-term lamivudine therapy," Gastroenterology, vol. 124, no. 1, pp. 105-117, 2003.

[38] Y.-F. Liaw, J. J. Y. Sung, W. C. Chow et al., "Lamivudine for patients with chronic hepatitis B and advanced liver disease," The New England Journal of Medicine, vol. 351, no. 15, pp. 15211587, 2004.
[39] A. Matsumoto, E. Tanaka, A. Rokuhara et al., "Efficacy of lamivudine for preventing hepatocellular carcinoma in chronic hepatitis B: a multicenter retrospective study of 2795 patients," Hepatology Research, vol. 32, no. 3, pp. 173-184, 2005.

[40] M.-F. Yuen, W.-K. Seto, D. H.-F. Chow et al., "Long-term lamivudine therapy reduces the risk of long-term complications of chronic hepatitis B infection even in patients without advanced disease," Antiviral Therapy, vol. 12, no. 8, pp. 12951303, 2007.

[41] S. J. Hadziyannis, N. C. Tassopoulos, E. J. Heathcote et al., "Long-term therapy with Adefovir Dipivoxil for HBeAgnegative chronic hepatitis B for up to 5 years," Gastroenterology, vol. 131, no. 6, pp. 1743-1751, 2006.

[42] E. R. Schiff, S. S. Lee, Y.-C. Chao et al., "Long-term treatment with entecavir induces reversal of advanced fibrosis or cirrhosis in patients with chronic hepatitis B," Clinical Gastroenterology and Hepatology, vol. 9, no. 3, pp. 274-276, 2011.

[43] T.-T. Chang, Y.-F. Liaw, S.-S. Wu et al., "Long-term entecavir therapy results in the reversal of fibrosis/cirrhosis and continued histological improvement in patients with chronic hepatitis B," Hepatology, vol. 52, no. 3, pp. 886-893, 2010.

[44] C.-C. Yeh, J.-T. Lin, L.-B. Jeng et al., "Nonsteroidal antiinflammatory drugs are associated with reduced risk of early hepatocellular carcinoma recurrence after curative liver resection: a nationwide cohort study," Annals of Surgery, vol. 261, no. 3, pp. 521-526, 2015. 


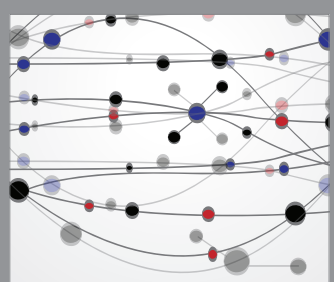

The Scientific World Journal
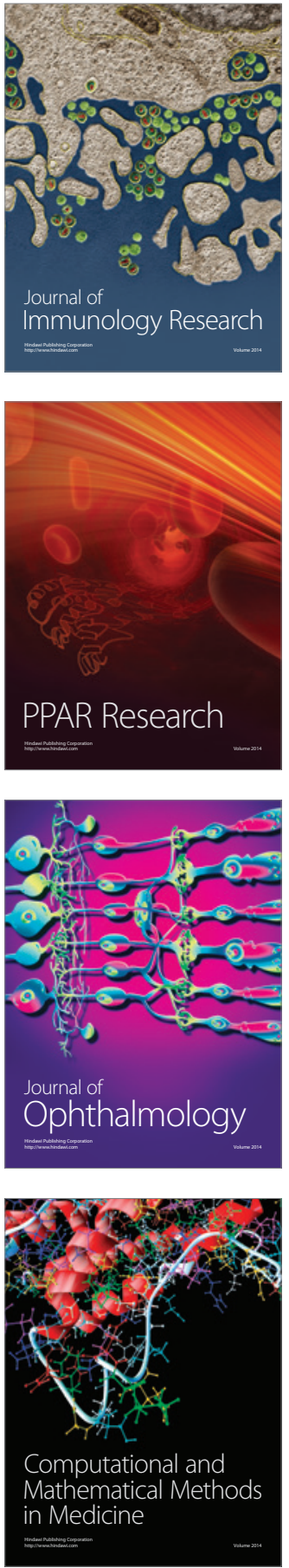

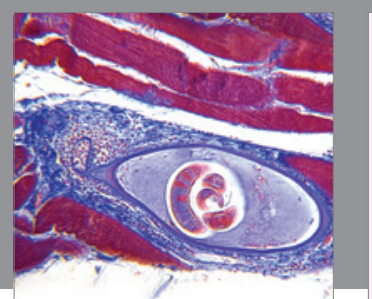

Gastroenterology Research and Practice

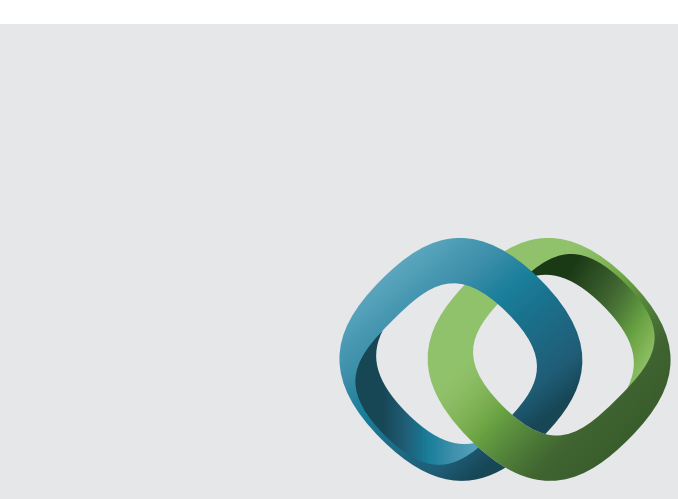

\section{Hindawi}

Submit your manuscripts at

http://www.hindawi.com
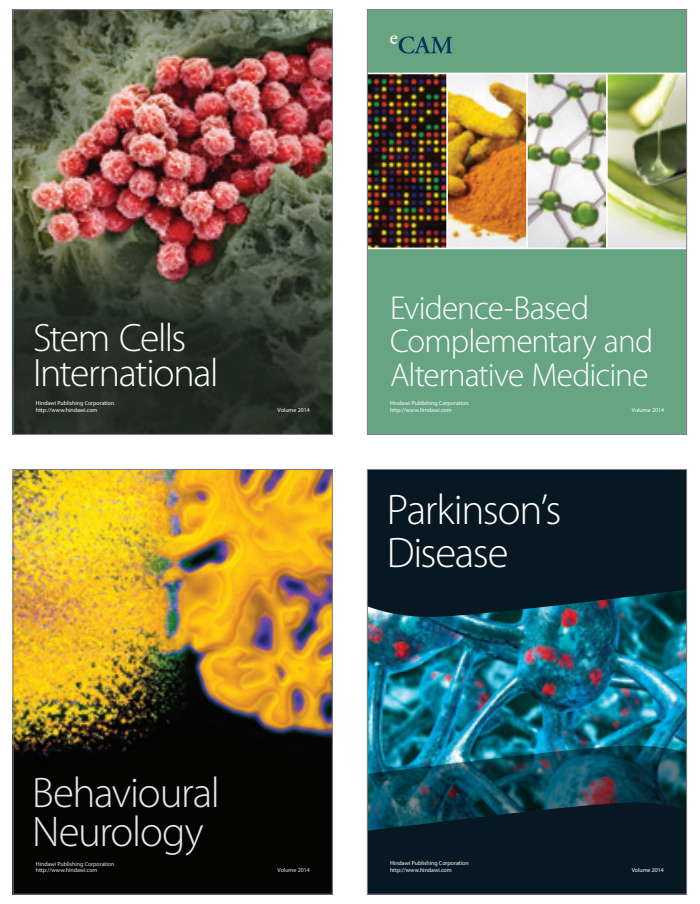


Disease Markers
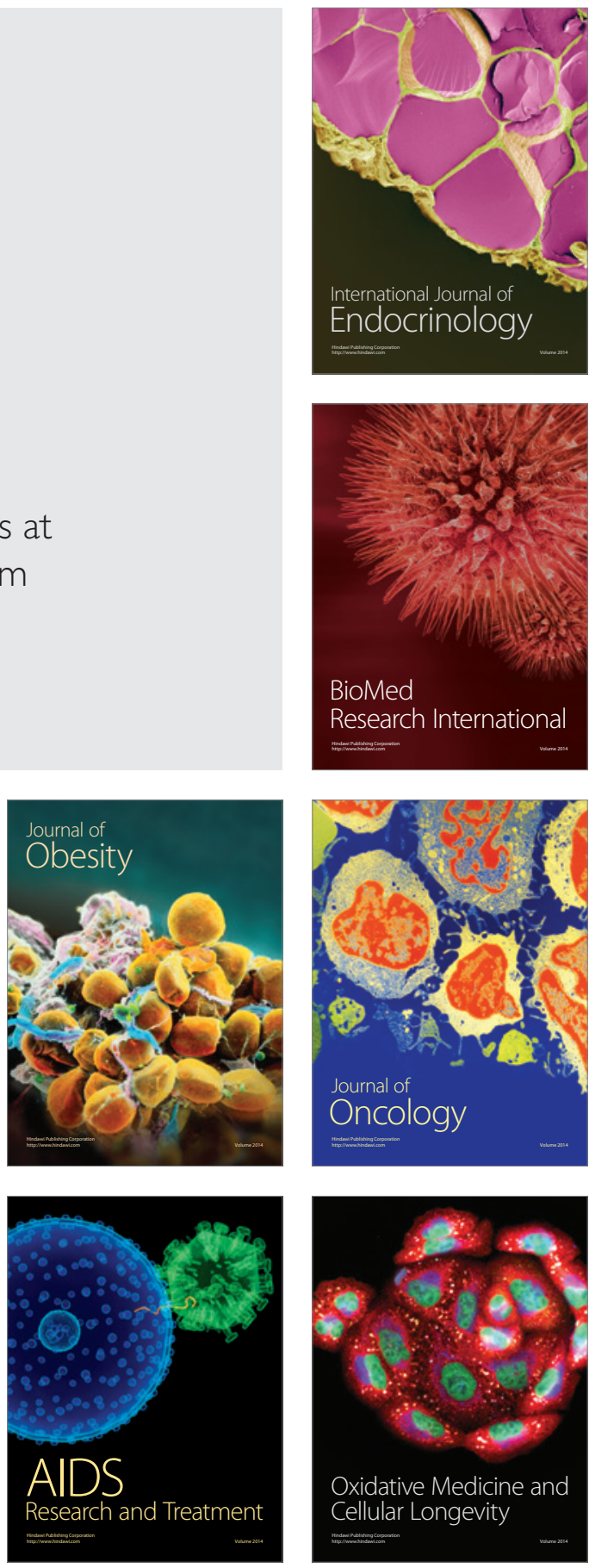